\title{
Empoderamiento y logros de aprendizaje en estudiantes mujeres de la Facultad de Ciencias Matemáticas
}

\author{
Carlos Alberto Peña Miranda ${ }^{1}$ y Elizabeth Cosi Cruz ${ }^{2}$
}

Resumen: El objetivo del presente trabajo de investigación fue determinar si el empoderamiento influye en los logros de aprendizaje, en una muestra de estudiantes mujeres de las cuatro escuelas profesionales que conforman la Facultad de Ciencias Matemáticas de la Universidad Nacional de San Marcos. Se utilizó un diseño correlacional causal, con una muestra de 200 estudiantes mujeres, a quienes se les aplicó el inventario de empoderamiento de Hernández, J. y García, R. (2008) y para los logros de aprendizaje se tomaron como datos, las notas finales que obtuvieron en el semestre académico 2017-II. Este instrumento fue sometido a los análisis respectivos que determinaron que la prueba es válida y confiable. Los resultados obtenidos indican que el empoderamiento influye en los logros de aprendizaje de las estudiantes de la muestra. También se comprobó la existencia de correlaciones entre cada uno de los factores del empoderamiento con los logros de aprendizaje.

Palabras clave: empoderamiento femenino; logros de aprendizaje; investigación correlacional.

\section{Empowerment and learning achievements in female students of the Faculty of Mathematical Sciences}

\begin{abstract}
The objective of this research was to determine if empowerment influences learning achievements in a sample of female students from the four professional schools that make up the Mathematical Sciences Faculty of the National University of San Marcos. A causal correlational design was used, with a sample of 200 female students, to whom the empowerment inventory of Hernández, J. and García, R. (2008) was applied and for the learning achievements were taken as data, the notes finals that obtained in the academic semester 2017- II. This instrument was subjected to the respective analyses that determined that the test is valid and reliable. The results obtained indicate that empowerment influences the learning achievements of the students in the sample. The existence of correlations between each of the factors of empowerment and learning achievements was also verified.
\end{abstract}

Keywords: female empowerment; learning achievements; correlational research.

Recibido: 20/07/2018. Aceptado: 18/11/2018. Publicado online: 31/12/2018.

(C)Los autores. Este artículo es publicado por la Revista PESQUIMAT de la Facultad de Ciencias Matemáticas, Universidad Nacional Mayor de San Marcos. Este es un artículo de acceso abierto, distribuido bajo los términos de la licencia Creative Commons Atribucion-No Comercia-Compartir Igual 4.0 Internacional.(http://creativecommons.org/licenses/by-nc-sa/4.0/) que permite el uso no comercial, distribución y reproducción en cualquier medio, siempre que la obra original sea debidamente citada. Para información, por favor póngase en contacto con revistapesquimat.matematica@unmsm.edu.pe

\footnotetext{
${ }^{1}$ UNMSM, Facultad de Ciencias Matemáticas. e-mail: cpenam@unmsm.edu.pe

${ }^{2}$ Universidad Privada Norbert Wiener, e-mail: elizabeth.cosi@uwiener.edu.pe
} 


\section{Introducción}

El empoderamiento es una herramienta muy útil en la actualidad, genera un sentimiento de bienestar en las personas y el poder implementarlo en una institución pública es de gran utilidad, ya que en la mayoría de ellas la opinión de las estudiantes no es tomada en cuenta, lo que les ocasiona una sensación de incapacidad. Incluso las estudiantes puede llegar a pensar que no son competentes, y el docente puede perder el aporte tan valioso que ellas puedan brindar. $\mathrm{Al}$ respecto, Bacqué y Biewener (2014) señala que el empoderamiento articula dos dimensiones, la del poder, que constituye la raíz de la palabra, y la del proceso de aprendizaje para acceder a éste.

Chiavenato (2009), por su parte, identifica el empoderamiento como poder, autoridad y responsabilidad a las personas para que sean más activas y proactivas dentro de la organización. En ese sentido, el docente universitario que forma a los futuros profesionales se le plantea el reto de potenciar su confianza para ser creativo, innovador y competente para que las estudiantes integre sus logros de aprendizaje en sus tres dimensiones, cognitivos, procedimentales y actitudinales.

Hernández, J. y García, R. (2008) han desarrollado un instrumento para medir el empoderamiento de la mujer clasificado en siete factores (empoderamiento participativo, temeridad, influencias externas, independencia, igualdad, satisfacción social y seguridad) donde se determi-na en qué medida y con que características se daba el proceso del empoderamiento de la mujer.

Por otro lado, los autores, también hace énfasis que el instrumento es un cuestionario que consta de 34 reactivos tipo Likert, diseñado para evaluar el empoderamiento de la mujeres. Puede aplicarse a mujeres de 15 a 73 años tanto en forma individual como grupal y su calificación es sencilla, no tiene un límite de tiempo establecido, pero se recomienda un tiempo de aplicación de 30 minutos aproximadamente.

En nuestro país, Varas, C. (2016) analizó la validez y confiabilidad del instrumento desarrollado por Hernández, J. y García, R. (2008). La muestra estuvo conformada por 399 mujeres profesionales de 22 a más años, con diferentes años de experiencia y estado civil. Los resultados estadísticos a que fue sometida la prueba demostraron que la prueba tiene validez y confiabilidad estadística, lo que hace posible su empleo en mujeres profesionales de Lima - Perú.

El presente trabajo fue desarrollado en la Facultad de Ciencias Matemáticas con el objetivo de comprobar si el empoderamiento influye en los logros de aprendizaje de las estudiantes mujeres de la Facultad de Ciencias Matemáticas.

\section{Metodología}

El método de la investigación es el método descriptivo y su diseño es correlacional causal ${ }^{1}$, se realizó en base a la información recolectada en la Facultad de Ciencias Matemáticas durante el semestre académico 2017-II.

La población estudiada estuvo conformada por las estudiantes mujeres de la Facultad de Ciencias Matemáticas que se matricularon durante el semestre académico 2017-II. Dicha población se dividió en cuatro estratos, según la escuela profesional (EP). En la Tabla 1 se puede apreciar que los estudiantes están distribuidos de acuerdo a la escuela profesional de pertenencia, notándose que la EP de Investigación Operativa tiene el mayor porcentaje (42.4\%).

\footnotetext{
${ }^{1}$ Estos diseños describen relaciones entre dos o más categorías, conceptos o variables en un momento determinado. (Hernández et al, 2010, p. 154)
} 
Tabla 1. Distribución de la población femenina por EP

\begin{tabular}{|c|c|c|}
\hline EP & Frecuencia & Porcentaje \\
\hline Matemática & 62 & 16,6 \\
\hline Estadística & 107 & 28,6 \\
\hline Investigación Operativa & 158 & 42,4 \\
\hline Computación Científica & 47 & 12,6 \\
\hline Total & 374 & 100,0 \\
\hline
\end{tabular}

Para determinar una muestra representativa de las estudiantes mujeres de la Facultad de Ciencias Matemáticas, que deberán ser incorporados en el estudio, se determinó con la fórmula²

$$
N=\frac{n G^{2} P Q}{E^{2}(n-1)+G^{2} P Q}
$$

Obteniéndose como muestra representativa 200 estudiantes mujeres cuyas edades fluctúan entre 20 y 26 años.

Para la recolección de datos se empleó como instrumento el inventario de empoderamiento de Hernández, J. y García, R. (2008) y para los logros de aprendizaje se tomaron como datos, las notas finales que obtuvieron las estudiantes mujeres en el semestre académico 2017-II. Una vez aplicado el instrumento de recolección, se realizó la digitación y procesamiento de la información obtenida, utilizando el software estadístico SPSS (Statistical Package for Social Sciences) que permitió y facilitó presentar los datos obtenidos en tablas.

\section{Resultados}

\subsection{Análisis de confiabilidad de la prueba de empoderamiento}

En la tabla 2 se puede observar que el análisis de la confiabilidad por consistencia interna a través del coeficiente Alfa de Cronbach asciende a 0,90, lo que permite concluir que la prueba de empoderamiento presenta confiabilidad.

Tabla 2. Análisis de confiabilidad de la prueba de empoderamiento

\begin{tabular}{|c|c|c|c|}
\hline Factores del empoderamiento & Media & D. E. & ritc \\
\hline Empoderamiento participativo & 15,35 & 2,39 & 0,59 \\
\hline Temeridad & 15,23 & 2,48 & 0,59 \\
\hline Influencias externas & 18,92 & 2,69 & 0,70 \\
\hline Independencia & 22,89 & 3,17 & 0,76 \\
\hline Igualdad & 26,03 & 3,33 & 0,74 \\
\hline Satisfacción social & 15,14 & 2,36 & 0,62 \\
\hline Seguridad & 14,81 & 2,22 & 0,63 \\
\hline \multicolumn{2}{|c|}{ Alfa de Cronbach $=0,90 *$} \\
\hline
\end{tabular}

${ }^{*} p<0,05^{* *} p<0,01^{* * *} p<0,001$

$N=200$

${ }^{2}$ Sierra Bravo, 1995, p. 191 


\subsection{Análisis de Validez de la prueba de empoderamiento}

En la tabla 3 observamos que la medida de adecuación del muestreo de Kaiser-Meyer-Olkin alcanza un valor de 0,90 que puede considerarse como adecuado, mientras que el test de esfericidad de Bartlett presenta un valor que es significativo, lo que nos indica que los coeficientes de correlación entre los factores del empoderamiento son lo suficiente elevados como para continuar con el análisis factorial.

También aprecia que existe un solo factor que explica el 75,57\% de la varianza total. Estos hallazgos nos permiten indicar que la prueba de empoderamiento presenta validez de constructo.

Tabla 3. Análisis de la validez de la prueba de empoderamiento

\begin{tabular}{|c|c|c|c|}
\hline Factores del empoderamiento & Media & D. E. & ritc \\
\hline Empoderamiento participativo & 15,35 & 2,39 & 0,69 \\
\hline Temeridad & 15,23 & 2,48 & 0,70 \\
\hline Influencias externas & 18,92 & 2,69 & 0,79 \\
\hline Independencia & 22,89 & 3,17 & 0,83 \\
\hline Igualdad & 26,03 & 3,33 & 0,82 \\
\hline Satisfacción social & 15,14 & 2,36 & 0,73 \\
\hline Seguridad & 14,81 & 2,22 & 0,073 \\
\hline Varianza Explicada & $75,57 \%$ \\
\hline Medida de adecuación del muestreo de Kaiser-Meyer-Olkin $=0,90$ \\
\hline Test de Esfericidad de Bartlett $=622,268{ }^{* * *}$ \\
\hline${ }^{*} p<0,05^{* *} p<0,01^{* * *} p<0,001$ \\
$N=200 \quad 0$
\end{tabular}

\subsection{Análisis de regresión}

Los resultados presentados en la tabla 4 indican que la correlación múltiple ${ }^{3}$ asciende a 0,62, la cual da una $\mathrm{R}$ cuadrado igual a 0,38 y que al ser corregida llega a 0,36 , lo cual indica que las variables predictoras permiten explicar el $37,6 \%$ de la varianza de la variable predicha que en este caso corresponde a logros de aprendizaje.

Tabla 4. Resumen del modelo de regresión múltiple de logros de aprendizaje

\begin{tabular}{|c|c|c|c|}
\hline Modelo & $\mathrm{R}$ & $\mathrm{R}$ cuadrado & $\mathrm{R}$ cuadrado corregida \\
\hline 1 & $0,618(\mathrm{a})$ & 0,376 & 0,358 \\
\hline
\end{tabular}

a. Variables predictoras: (Constante), Empoderamiento participativo, satisfacción Social, temeridad, influencias externas, inde-pendencia, igualdad y seguridad.

b. Variable dependiente: Logros de aprendizaje

También se puede aprecia en la tabla 5 el análisis de varianza indica que se alcanza un estadístico $\mathrm{F}=16,856, \mathrm{p}=0,000$ el cual es significativo. Este resultado permite concluir que la ecuación de regresión es estadísticamente significativa y permite predecir de forma consistente la variable predicha o dependiente, en este caso logros de aprendizaje.

\footnotetext{
${ }^{3} \mathrm{Al}$ grado de relación que existe entre tres o más variables se le conoce como correlación múltiple. (Spiegel y Stephens, 2009, p. 384)
} 
Tabla 5. Análisis de varianza de la ecuación de regresión múltiple de logros de aprendizaje

\begin{tabular}{|c|c|c|c|c|c|c|}
\hline Modelo & & Suma de cuadrados & gl & Media cuadrática & F & Sig. \\
\hline & Regresión & 99,651 & 7 & 14,237 & 16,856 & $0,000^{b}$ \\
\hline 1 & Residual & 163,026 & 193 & 0,845 & & \\
\hline & Total & 262,672 & 200 & 0,845 & & \\
\hline
\end{tabular}

a. Variables predictoras: (Constante), Empoderamiento participativo, satisfacción Social, temeridad, influencias externas, independencia, igualdad y seguridad.

b. Variable dependiente: Logros de aprendizaje

\subsection{Análisis de Correlación}

En la tabla 6 observamos los resultados entre los siete factores de la variable empoderamiento con la variable logros de aprendizaje, el estadístico usado es el rho de Spearman, el mismo que indica que existen correlaciones significativas entre las variables de estudio.

Tabla 6. Análisis de correlación entre los factores del empoderamiento y los logros de aprendizaje

\begin{tabular}{|c|c|}
\hline Factores del empoderamiento & Logros de aprendizaje \\
\hline Empoderamiento participativo & $0,56^{* * *}$ \\
\hline Temeridad & $0,50^{* * *}$ \\
\hline Influencias externas & $0,58^{* * *}$ \\
\hline Independencia & $0,60^{* * *}$ \\
\hline Igualdad & $0,68^{* * *}$ \\
\hline Satisfacción social & $0,52^{* * *}$ \\
\hline Seguridad & $0,50^{* * *}$ \\
\hline
\end{tabular}

${ }^{*} p<0,05^{* *} p<0,01^{* * *} p<0,001$

$N=200$

\section{Discusión de los resultados}

Los resultados obtenidos y que se expresan en las Tablas 4 y 5 indican que en efecto la variable empoderamiento está afectando significativamente los logros de aprendizaje, por lo que podemos afirmar que "el empoderamiento influye significativamente en los logros de aprendizaje de las estudiantes mujeres de la Facultad de Ciencias Matemáticas".

Así mismo podemos señalar que estos resultados se encuentran relacionados a los resultados encontrados por Andrade, C. (2014), Ramírez, S.(2017) y Rocha, L. (2011), quienes resaltan la importancia del empoderamiento femenino en diversos aspectos de la vida de las mujeres como por ejemplo la identidad de genero y el aprendizaje colaborativo.

\section{Conclusiones}

Luego de analizar los datos obtenidos entre las variables: empoderamiento y logros de aprendizaje, se determina que el empoderamiento influye significativamente en los logros de aprendizaje de las estudiantes mujeres y afianzando el objetivo principal de la investigación. 
Después de analizar la relación que existe entre los factores del empoderamiento frente a los logros de aprendizaje de las estudiantes mujeres, se determinó que existe una correlación moderada y positiva entre cada una de los factores del empoderamiento con los logros de aprendizaje.

\section{Referencias bibliográficas}

[1] Andrade, C. (2014). Relación de identidad de genero y empoderamiento en un grupo de mujeres (tesis de maestría). Universidad Iberoamericana, México D.F, México.

[2] Bacqué, M. y Biewener, C. (2013). El empoderamiento, una práctica emancipadora. Buenos aires, Argentina: Editorial Gedisa.

[3] Chiavenato, I. (2011). Adminiistración de recursos humanos. México: Mc Graw Hill.

[4] Hernández, R., Fernández, C. y Baptista, P. (2010). Metodología de la Investigación. México: Mc Graw Hill.

[5] Hernández, J. y García, R.(2008). Instrumento para medir el empoderamiento de la mujer. Villahermosa, Tabasco. México: Universidad Juárez Autónoma de Tabasco. Recuperado de http://www.archivos.ujat.mx/2011/difusion/libros/10.pdf

[6] Rocha, L. (2011). Empoderamiento de mujeres divorciadas. Trabajo de Investigación para obtener el Diploma de Estudios Avanzados. Universidad Pablo de Olavide, Sevilla, España.

[7] Sierra, B. R. (1995). Técnicas de Investigación Social, Teoría y Ejercicios. Madrid: España: Parainfos.

[8] Spiegel, M. y Stephens, L. (2009). Estadística. México: Mc Graw Hill.

[9] Varas, C. (Septiembre de 2018). Propiedades psicométricas del instrumento para la medición del empoderamiento IMEM, aplicado en mujeres profesionales, 2016. VII Congreso Internacional de Psicología y Educación 2018, Santa Marta Colombia, Colombia. 